\title{
Predicting elongation efficiency of gene translation for annotation of bacterial genomes: a case study for biosynthetic gene clusters of nonribosomal peptides
}

\author{
A.I. Klimenko \\ Kurchatov Genomics Center, \\ ICG SB RAS \\ Novosibirsk, Russia \\ klimenko@bionet.nsc.ru
}

\author{
Yu.G. Matushkin \\ Institute of Cytology and Genetics, \\ ICG SB RAS \\ Novosibirsk, Russia
}

\author{
D.A. Afonnikov \\ Kurchatov Genomics Center, \\ ICG SB RAS, \\ Novosibirsk, Russia \\ Novosibirsk State University, NSU \\ Novosibirsk, Russia
}

\begin{abstract}
The gene expression levels for bacteria are largely determined by the efficiency of translation elongation. We have performed bioinformatic elongation efficiency analysis of NRP biosynthetic gene clusters (BGCs) obtained from ANTISMASH-DB using whole-genome sequences of bacterial genomes that are available at NCBI Genbank. The analysis has provided the information about distribution of nonribosomal peptide biosynthetic gene clusters in bacteria and their putative translation elongation efficiency.
\end{abstract}

Key words - nonribosomal peptides; translation elongation efficiency; bacteria; genome annotation

\section{Motivation and Aim}

It is hard to underestimate the crucial role that efficiency of gene expression plays in synthetic biology and genome engineering. Though being affected at several levels such as transcription, translation, posttranslational modification, etc., the gene expression levels for bacteria are largely determined by the efficiency of translation elongation [1]. Nonribosomal peptides (NRPs) constitute an important fraction of bacterial peptidomes acting as antibiotics, toxins, surfactants, siderophores, anti-tumor agents and immune response modifiers [2]. Biosynthesis of NRPs is dependent on particular enzymes - nonribosomal peptide synthetases (NRPSs), which are encoded by clusters of genes in bacterial genomes [3].

\section{Methods and Algorithms}

The EloE software [4] is a tool for gene ranking based on their putative translation elongation efficiency inferred from their nucleotide sequences taking into account such factors as codon composition, presence and stability of secondary structures in mRNA [1]. The obtained predicted values correlate with available experimental data on gene expression in different microorganisms [4]. Thus, EloE is useful as a bioinformatic tool for genome annotation that enables a researcher with a capacity to infer a priori estimates of gene expression efficiency based on whole-genome nucleotide sequences only. We have performed bioinformatic analysis of NRP biosynthetic gene clusters (BGCs) obtained from ANTISMASH-DB [5] using whole-genome sequences of bacterial genomes that are available at NCBI Genbank. The analysis is based on the method predicting gene translation elongation efficiency that is implemented in the EloE software [4]. Statistical and bioinformatic analysis scripts have been developed on Python using software library Biopython.

\section{Results}

The statistical analysis has revealed the taxa possessing genomes enriched by BGCs. The analysis of predicted efficiency of translation elongation has shown that while only $6.6 \%$ NRPS genes fall into the category of high predicted efficiency of translation, there is a number of BGCs distinguished by both large absolute number of genes with high predicted efficiency of translation and their percentage. These BGCs belong to such genera as Pseudomonas, Staphylococcus, Corynebacterium, Streptomyces, Amycolatopsis, Paenibacillus, Rhodococcus and Burkholderia.

\section{Conclusion}

The performed bioinformatic analysis has provided the information about distribution of nonribosomal peptide biosynthetic gene clusters in bacteria and their putative translation elongation efficiency. Thus, the analysis of translation elongation efficiency is useful as a high-throughput technique for gene ranking in bacterial genomes, which can be regarded as a rough estimate of expression level for various groups of genes of interest.

\section{ACKNOWLEDGMENT}

This study was funded by the Russian Foundation for Basic Research (Grant Nos. 17-00-00470 (K), 17-00-00462). The computational resources of the Joint HPC Facility 'Bioinformatics' was used with the support of the budget project No. 0324-2019-0040-C-01.

\section{References}

[1] V. A. Likhoshvai and Y. G. Matushkin, "Nucleotide compositionbased prediction of gene expression efficacy," Mol. Biol., vol. 34, no. 3, pp. 406-412, 2000.

[2] S. Caboche, M. Pupin, V. Leclère, A. Fontaine, P. Jacques, and G. Kucherov, "NORINE: A database of nonribosomal peptides," Nucleic Acids Res., vol. 36, no. SUPPL. 1, pp. 326-331, 2008.

[3] R. D. Süssmuth and A. Mainz, "Nonribosomal Peptide SynthesisPrinciples and Prospects," Angew. Chemie - Int. Ed., vol. 56, no. 14, pp. 3770-3821, 2017.

[4] V. Sokolov, B. Zuraev, S. Lashin, and Y. Matushkin, "Web application for automatic prediction of gene translation elongation efficiency.," $J$. Integr. Bioinform., vol. 12, no. 1, p. 256, 2015.

[5] K. Blin, M. H. Medema, R. Kottmann, S. Y. Lee, and T. Weber, "The antiSMASH database, a comprehensive database of microbial secondary metabolite biosynthetic gene clusters," Nucleic Acids Res., vol. 45, no. D1, pp. D555-D559, 2017. 